\title{
Effect of multi-domain structure on ionic transport, electrostatics, and current evolution in $\mathrm{BaTiO}_{3}$ ferroelectric capacitor
}

\author{
YeCao, ${ }^{\mathrm{a}, 1}$ JieShen, ${ }^{\mathrm{b}}$ CliveRandall and Long-Qing Chen ${ }^{\mathrm{a}}$ \\ ${ }^{\mathrm{a}}$ Department of Materials Science and Engineering, The Pennsylvania State University, University Park, PA 16802, USA \\ ${ }^{\mathrm{b}}$ Department of Mathematics, Purdue University, W Lafayette, IN 47907, USA
}

\begin{abstract}
The semiconducting properties of certain ferroelectric materials, partially caused by the interactionsamong ferroelectric polarizations, charged domain walls, and conducting point defects, pose a threat to the reliability of ferroelectrics used as dielectric material in capacitor devices, and is not yet fully understood.We proposed a physical model combining the phase-field method for ferroelectric domain structures and diffusion equations for defect transport to study the resistance degradation behavior in multi-domain tetragonalBaTiO 3 capacitors. We considered a hypotheticalNi/BaTiO $3 / \mathrm{Nisingle} \mathrm{parallel} \mathrm{plate} \mathrm{capacitor} \mathrm{configuration} \mathrm{subject} \mathrm{to} 0.5 \mathrm{~V}$ dc bias at $25^{\circ} \mathrm{C}$. It is found that $90^{\circ}$ domain walls are charged, induce local space charge segregation, and form local electric potential barriers upon external biasthat significantly influence the ionic transport behavior. Additionally, the $180^{\circ}$ domain walls remain nearly charge neutral and have much less influence on ionic transport. The effect of domain wall and polarization orientations on the leakage current evolutionis investigated. Our study could necessitate further understanding on the influence of ferroelectric state, ionic interactions, transport barriers, spatial distributions, and breakdown phenomena.
\end{abstract}

Keywords: Defect transport, $\mathrm{BaTiO}_{3}$, domain structure, resistance degradation, phase-field simulation

\footnotetext{
${ }^{1}$ Corresponding author. Tel.: +1 (814)-876-0910. E-mail: yxc238@psu.edu
} 


\section{Introduction}

Ferroelectric oxides, such as barium titanite $\left(\mathrm{BaTiO}_{3}\right)$, have been extensively used as dielectric materialsinmulti-layer ceramic capacitors (MLCCs) in various electronic devices[1].The trend of further miniaturization of MLCC devices for high capacitance resulted in ever-decreasing dielectric thickness, down to sub-micron levels, and ever-increasing electric field strength distributed across each dielectric layer under given voltage stresses[2].This causes a major challenge tothe reliability of MLCCs, among which the long-term resistance degradation is one of the primary limiting factors to the life time of MLCCs. The long-term resistance degradation is characterized by slowly increasing leakage currents undera DC biaswell below the critical breakdown voltage[3].

In the dielectric industry, the evaluations of resistance degradation, and thus the longterm reliability of MLCCs,are generally accessed by an engineering approach called the highly accelerated lifetime tests (HALTs)[3, 4]. This method evaluates the lifetime of MLCCsby extrapolatingtheHALTsdata measured under various temperatures and field stresses to the real working condition based on the Erying model[5]. Although this method shows advantages in accelerating the measurement at high temperature and voltage stress in paraelectric phase, it rules out the possible influence of complex ferroelectric domain structures and the associated piezoelectric strains on the degradation processof ferroelectric-based capacitor. Therefore, the HALTs evaluated long-term reliability accessed in the paraelectricphase may not represent the real lifetime of ferroelectric capacitors.

On the other hand, it is of scientific importance to understand the mechanism of the ion transport and its relationship to resistance degradation. Over the past 20 years, it has been 
investigated by a number of experimental approaches, including the electro-coloration measurement[6, 7],impedance spectroscopy (IS)[8],thermal simulated depolarization current (TSDC) $[9,10]$, transmission electron microscopy (TEM)[11], and theoretical calculations, such as finite element method[12-14]. While it is generally recognized that the oxygen vacancy migration plays an important role in the degradation process, it is still unclear how the ferroelectric domain structurescould influence the ionic/electronic distribution and transport behavior. The authors have recently studied the effect of ferroelectric polarization on resistance degradation behavior in single domain $\mathrm{BaTiO}_{3}$ capacitor[15]; however,a single domainconfiguration is rarely formed in real $\mathrm{BaTiO}_{3}$ capacitors. It wasreported that domain walls contribute to thedielectric and piezoelectric properties of ferroelectric materials[16, 17] and could pin the local space charge and affect the transport behavior due to the polarization charges [18-21] and mechanical stresses $[22,23]$ developed along ferroelastic domain walls. Therefore, to obtain a more complete fundamental understanding of resistance degradationbehavior in ferroelectric capacitor,a physics-based theoretical model capable of incorporating the multidomain structure and defect transport interaction is imperative.

In this work,we developed a combined phase-field and transport model to understand the role of ferroelectric domain and domain walls on ionic transport and resistance degradation behavior, and to estimate the long-term reliability ofreal ferroelectric based capacitor. The phasefield method has been successfully applied to studying the ferroelectric domain structures in both bulk and thin film systems [24-28].In this study, we chose the sandwiched $\mathrm{Ni} / \mathrm{BaTiO}_{3} / \mathrm{Ni}$ single parallel plate capacitor configuration as the model system. The effects of ferroelectric/metal interfaces and domain wall orientations on the ionic segregation/depletion, local electric potential and field distribution,and ionic migration upon external field and leakage current evolution have 
been extensively studied.Our work provides some fundamentalinsights intothe complex interactions among ferroelectric domain structure, defect transport and dielectric degradation behavior.

\section{Model}

In the phase-field model of multi-domainBaTiO${ }_{3}$ capacitor, the ferroelectric system is represented by choosing the polarization vector $P_{i}=\left(P_{1}, P_{2}, P_{3}\right)$ as the order parameter to describe the ferroelectrics domain structures. The domain evolution is modeled by the temporal evolution of $P_{i}$ obtained by minimizing the total free energy of the system with respect to $P_{i}$,

$$
\frac{\partial P_{i}(\boldsymbol{r}, t)}{\partial t}=-L \frac{\delta F}{\delta P_{i}(\boldsymbol{r}, t)},(i=1 \sim 3)
$$

in which $t$ is the time step and $L$ is the kinetic coefficient related to domain mobility. The total free energy $\mathrm{Fof}_{\mathrm{BaTiO}}$ single crystal is expressed as,

$$
F=\int_{V}\left[f_{\text {lan }}\left(P_{i}\right)+f_{\text {grad }}\left(P_{i, j}\right)+f_{\text {elas }}\left(P_{i}, \varepsilon_{i j}\right)+f_{\text {elec }}\left(P_{i}, E_{i}\right)\right] d V
$$

where $V$ is the system volume, $\varepsilon_{i j}$ and $E_{i}$ denote the components of strain and electric field, $P_{i, j}=\partial P_{i} / x_{j}$, and $f_{\text {lan }}\left(P_{i}\right), f_{\text {grad }}\left(P_{i, j}\right), f_{\text {elas }}\left(P_{i}, \varepsilon_{i j}\right)$ and $f_{\text {elec }}\left(P_{i}, E_{i}\right)$ representthe LandauDevonshire free energy density, the gradient energy density, and elastic energy density and the electrostatic energy density, respectively. Detailed expressions of each of the local free energy densities can be found in literature[25, 26].

The charge defect distribution, transport, and blocking at electrodes play an important role in resistance degradation phenomena. In this study, the intrinsic and extrinsic major 
chargedefects of interestinclude the oxygen vacancies $\left(V_{O}^{\bullet \bullet}\right)$, electrons $\left(e^{\prime}\right)$, holes $\left(h^{\bullet}\right)$, and ionized acceptors $\left(A^{\prime}\right)$ written in Kröger-Vink notation[29]. The +2 oxygen vacancies are created during high temperature annealing of capacitors, and their concentration is assumed to be conserved at the temperature when degradation measurements are conducted. The acceptors are introduced by substitution of +3 elements, such as $\mathrm{Fe}$ and $\mathrm{Al}$, on the $+4 \mathrm{Ti}$ sites $\left(\mathrm{Fe}_{\mathrm{Ti}}^{\prime}\right.$ and $\mathrm{Al}_{\mathrm{Ti}}^{\prime}$ $\left.=A^{\prime}\right)$ and are considered fully ionized and immobile.

We assumed in the simulation that all the charged carriers are homogeneously distributed in the entire $\mathrm{BaTiO}_{3}$ layer, and local charge neutrality condition is satisfied in the initial state,

$$
\rho(x)=\sum_{i} z_{i} e_{0} c_{i}(x)=0 \quad\left(i=V_{O}^{\bullet \bullet}, p, n, A^{\prime}\right)
$$

where $\rho(x)$ is the space charges at positon $x, e_{0}$ is the unite charge, $c_{i}$ and $z_{i}$ are the concentration and charge number of given species $i .\left(z_{V_{0}^{*}}=+2, z_{h^{\circ}}=+1\right.$ and $\left.z_{e^{\prime}}=z_{A^{\prime}}=-1\right)$ The migrations of the mobile charge defects are described by solving the linear transport equations, considering only concentration gradient and local electric field as the driving forces,

$$
\begin{aligned}
& \vec{J}_{i}=-D_{i} \vec{\nabla} c_{i}+z_{i} \mu_{i} c_{i} \vec{E} \\
& \frac{\partial c_{i}}{\partial t}=-\vec{\nabla} \bullet \vec{J}_{i}
\end{aligned}
$$

in which $J_{i}, D_{i}, \mu_{i}$ denote the flux, diffusivity, and mobility of given charge speciesi. $\vec{\nabla}=\frac{\partial}{\partial x} \vec{i}+\frac{\partial}{\partial y} \vec{j}+\frac{\partial}{\partial z} \vec{k}$ is the gradient operator. $\vec{E}$ is the local electric field component. We assume that the plate electrodesform blocking contact to the oxygen vacancy transport, thus the boundary condition of Eq. (4) for oxygen vacancy is specified as, 


$$
\vec{J}_{V_{o}^{* *}}=0
$$

On the other hand, the mobility of electrons and holes are several orders of magnitude higher than that of oxygen vacancy. They are assumed to reach steady state for each oxygen vacancy evolution. Mathematically we solve the steady state equations for electron $(\partial n / \partial t=0)$ and hole evolution $(\partial p / \partial t=0)$. The electronic concentrations at the metal/ferroelectric interfaces are fixed by the work function of the metal electrode (Ni). The boundary conditions of Eq. (4) for electrons and holes are written as,

$$
\begin{aligned}
& n_{z=0 . L}=N_{c} \exp \left(-\frac{E_{c}-E_{f m}}{k_{B} T}\right) \\
& p_{z=0 . L}=N_{v} \exp \left(-\frac{E_{f m}-E_{v}}{k_{B} T}\right)
\end{aligned}
$$

in which $N_{c}$ and $N_{v}$ are the effective density of states in the conduction and valence band of $\mathrm{BaTiO}_{3}$, where $E_{\mathrm{c}}$ and $E_{\mathrm{v}}$ represent the energy of the conduction band minimum and valence band maximum. $E_{f m}$ is the work function of plate electrode, and $k_{B}$ is the Boltzmann constant.

The interaction between electric potential, space charge, and polarization induced bound charge are described by the electrostatic equilibrium equation considering the space charge distribution,

$$
D_{i, i}=D_{1,1}+D_{2,2}+D_{3,3}=\rho
$$

in which $D_{i, i}=\partial D_{i} / \partial x_{i}$ while $D_{i}$ is the electric displacement, which is related to the electric field and polarization component through, 


$$
D_{i}=\kappa_{0} \kappa_{i j} E_{j}+P_{i}(i=1 \sim 3)
$$

in which $\kappa_{0}$ is the vacuum permittivity and $\kappa_{i j}$ is the dielectric constant. The polarization induced charges are introduced by $-P_{i, i}=-\left(P_{1,1}+P_{2,2}+P_{3,3}\right)$, and the electric field $\vec{E}$ is related to the electric potential through,

$$
E_{i}=-\phi_{, i}(i=1 \sim 3)
$$

Combining Eqs. (7) (9) and assuming $\kappa_{i j}=0(i \neq j)$, the electrostatic equilibrium equation (Eq. (7)) can be rewritten as,

$$
\kappa_{11} \phi_{, 11}+\kappa_{22} \phi_{, 22}+\kappa_{33} \phi_{, 33}=\frac{1}{\kappa_{0}}\left(P_{1,1}+P_{2,2}+P_{3,3}-\rho\right)(10)
$$

The boundary condition for Eq. (10) is defined as,

$$
\left.\phi\right|_{z=0}=V_{a}(0),\left.\quad \phi\right|_{z=L}=V_{a}(L)
$$

inwhich $V_{a}(0)$ and $V_{a}(L)$ are specified from the externally applied potential bias at $z=0, L$.

To model the polarization induced bound charges at the $\mathrm{Ni} / \mathrm{BaTiO}_{3}$ interfaces, we specify the polarization distribution at the boundaries as,

$$
a \times P_{i}-b \times\left.\frac{d P_{i}}{d z}\right|_{z=0}=0, \quad a \times P_{i}+b \times\left.\frac{d P_{i}}{d z}\right|_{z=L}=0(i=1 \sim 3)
$$

where the coefficients $a$ and $b$ are chosen to be 1.0 and $0.05 \mathrm{~cm}$ to represent the case where the charges at the metal/ferroelectric interfaces are partially compensated . 
The current density is contributed from the flow of mobile charged defects,including oxygen vacancies, electrons, and holes. The current density for particular charged carrier $i$ and the total current density are given by,

$$
\begin{aligned}
& I_{i}=Z_{i} e_{0} J_{i} \\
& I_{\text {total }}=\sum_{i} I_{i}
\end{aligned}
$$

whereionly include mobile species $\left(i=V_{o}^{\bullet \bullet}, e^{\prime}, p^{\bullet}\right)$.

\section{Simulation results and discussion}

\subsection{Initial Setup}

We started with a preset 2 Dmulti-domain tetragonal $\mathrm{BaTiO}_{3}$ as the model system, which consists offour possible domain variants, i.e., $a+(100), a-(-100), c+(001)$, andc-(00-1) in a headto-tail configurationto formboth $180^{\circ}$ and $90^{\circ}$ domain walls (Fig. 1(a)). The $\mathrm{BaTiO}_{3}$ film is $500 \mathrm{~nm}$ in thickness and is constrained by Ni plate electrodesof normal vector along $z(001)$. The external applied bias is along $+z$ direction. We set up a coordinate system withperiodic boundary condition along $x(100)$ direction andnon-periodic general boundary condition along $z(001)$ direction. The simulation size is discretized intoa 2D mesh of $400 \Delta x \times 400 \Delta z$, while $\Delta z$ is nonuniform. The temporal evolution of the polarizations and defect concentrationsare obtained by solving the TDGL equations and transport equations using the combined semi-implicit Fourier spectral method[30] and Chebyshev collocation method[31]. The material constants and energy coefficients aretaken from the literature[28].The parameters used in the simulation are listed in Table 1. 


\subsection{Equilibrium state}

We first evolved the preset domain structureunder room temperature without an external bias $\left(\mathrm{U}_{\mathrm{DC}}=0\right)$ toreach equilibriumstate, in which both $90^{\circ}$ and $180^{\circ}$ domain walls becomestable and form astep-like domain configuration(Fig. 1(a)). It is clearly seen that the positive/negative alternating polarization charges are bounded to the $90^{\circ}$ domain walls, while the $180^{\circ}$ domain walls and the domain interior remain charge neutral (Fig. 1(b)). This is due to the gradual rotations of polarizations across $90^{\circ}$ domain walls of finite thickness. The $90^{\circ}$ charged domain walls are locally compensated by an ionic (oxygen vacancy) segregation (Fig. 1(c)). It should be noted that the electronic charge compensation is negligible,since electronic charges are assumed to be fixed at the metal/ferroelectric interfaces in the simulation, and consequently theirconcentrations are much lower than ionic charge carriers. The ionic charges do not fully compensate the polarization bound charges. This inducesa small internal electric potential even in the absence of external applied bias (Fig. 1(d)), and a negative shear stress $\left(\sigma_{5}\right)$ along the $90^{\circ}$ domain walls (Fig 1(e)). A 1-D plot of the charge distributions along particular directions (indicated by red and green dashed lines in (a)) clearly shows that only $90^{\circ}$ domain walls exhibit alternating polarization charges(dashed lines in (f)) and space charges (solid lines in (f)) (always in the opposite direction for compensation), with the latter being much smaller than the former in magnitude. The resulting net charges are between $-5.0 \sim 5.0 \mathrm{C} / \mathrm{cm}^{3}$ across the $90^{\circ}$ domain walls. Notably large polarization charges are also found at the metal/ferroelectric interfaces, where the polarizations are oriented perpendicular to the contacting plate electrodes (green dashed lines in (f), not shown in (b)). This is because the electrodes are assumed to partially compensate the polarization charges. When the polarizations are oriented parallel to the contacting electrodes, there are no interfacial charges induced (pink dashed lines in (f)). 


\subsection{Evolution under applied bias}

When the domain structure and the local charges reach an equilibrium state, a dc bias of $0.5 \mathrm{~V}$ is applied along $+z$ direction.Fig. 2 (a) (d) show the temporal evolution of oxygen vacancies along $\mathrm{z}$ directions across the center of each of the $1 / 4$ sections of the entire domain (the corresponding domain structures are shown in the insets). Under the applied bias, oxygen vacancies electromigrate towards the cathode side $(z / H=1.0)$, which results in ionic segregation in the vicinity of the cathode region and depletion in the anode region $(z / H=-1.0)$. It is also seen that part of the oxygen vacancies pile up in front of the $90^{\circ}$ domain walls and under these conditions are locally trapped and not free tomigrate towards cathode, due to the polarization charges along $90^{\circ}$ domain walls. This becomes obvious in those domains with local polarizations oriented along the transport direction $(+z)$, whereionic enrichment and depletion are both seen locally inside the domain.Such local ionic redistribution are also seen in domains of polarization orientation opposite to the transport direction (-z), but the extent of local enrichment/depletion is less than the previous case. On the other hand, $180^{\circ}$ domain walls are less, if not at all, chargedand have nearly no influence on the oxygen vacancy transport. Clearly, the polarizations along $+z$ directions tend to promote the oxygen vacancy transport, as is further evidenced by the maximum local oxygen vacancy accumulation of $\sim 10^{20} \mathrm{~cm}^{-3}$ found at the cathode region, where adjacent domain is along $+z$ (Fig. 2(c)) and agrees with previous report[15].

To further understand the interaction between the $90^{\circ}$ domain walls and ionic transport, we studied the electric potential and field distribution under $U_{D C}=0.5 \mathrm{~V}$. Figure 3 (a) $\sim(d)$ show the 1D electric potential (solid black lines) and electric field (solid red lines) distributionsacross the center of each of the $1 / 4$ sections of the entire domain at time $t=2.5 \times 10^{6} \mathrm{~s}$. The light blue arrows represent the domain orientations along $z$ directions, while the dashed lines indicate the 
domain wall positions. From Fig. 3, the electric potential drops are mainly seen in " $\rightarrow$ " oriented domains along the applied field direction. This also explains why oxygen vacancy transports are promoted in thoselocal domains(Fig. 2). The electric potential drops becomemuch smaller in

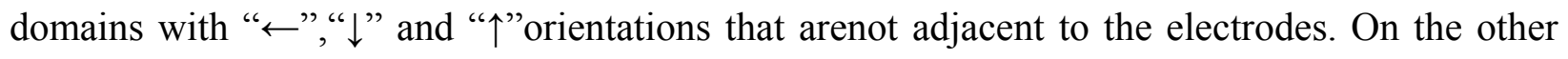
hand, remarkable electric potential gradients and local electric field enhancements can be found across the $90^{\circ}$ domain walls (indicated by red and green dashed lines). For “ $\downarrow \leftarrow$ ” and “ $\downarrow$ ”types of domain walls (indicated by green dashed lines), the drops of electric potential induce local positive electric fields of $\sim+100 \mathrm{kV} / \mathrm{cm}$. The oxygen vacancy drift under this positive field compensates oxygen vacancy diffusion in the opposite direction due to the local concentration gradient, which is induced by the polarization chargeslocated at the $90^{\circ}$ domain wall.This is further illustrated by a combined replot of Fig. 1(f), Fig. 2(b) and Fig. 3(b) into Fig. 4. For “个 $\rightarrow$ " and " $\rightarrow \uparrow$ ”types of domain walls (indicated by red dashed lines), local potential barriers are formed withnegative local electric fieldsto block the ionic transport. This causes local ionic accumulation and depletion on each side of the $90^{\circ}$ domain walls. The $180^{\circ}$ domain walls (indicated by blue dashed lines in Fig. 3) are less interacted with the electric potential distributions, and the electric fields are much smaller than those found at $90^{\circ}$ domain walls.

\subsection{Influence of domain structure on leakage current evolutions}

The effects of multi-domain structures on leakage current evolution in $\mathrm{Ni} / \mathrm{BaTiO}_{3} / \mathrm{Ni}$ single layer capacitor under DC bias are further investigated. For comparison, we considered four additional scenarios of $\mathrm{BaTiO}_{3}$ composed of: (1) single domain $\operatorname{ofP}_{\mathrm{z}}$ along $+\mathrm{z}$ direction (along the applied field direction), (2)single domain of $\mathrm{P}_{\mathrm{z}}$ along $-\mathrm{z}$ direction, (3) $90^{\circ}$ domain

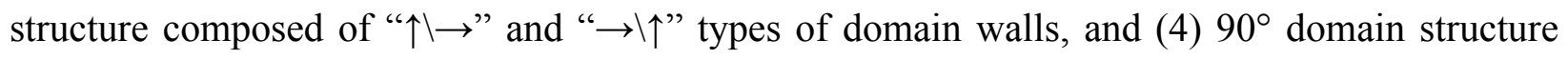

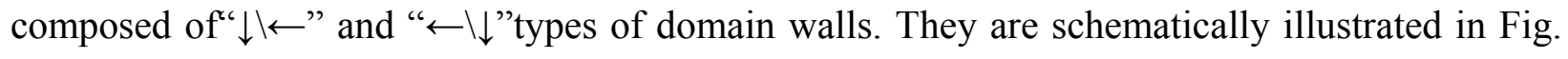


5(a), and the current density evolutions for scenario(1) (4) and multi-domain structure (5) are plotted and compared in Fig. 5(b). It is seen that the leakage currents increase slowly in the initial steps, followed by a fast and significant increase, which indicates the dielectric breakdown of capacitors. The sudden increases of leakage currents are attributed to the oxygen vacancy migrations and accumulations at the cathode regions. By comparing the degradation characteristic time $\left(t_{\mathrm{ch}}\right)$, which is defined as the critical time when leakage current increases $10 \times$ compared to its initial value (marked by "+" in Fig 5(b)), it is found that $t_{\text {ch }}$ is fastest in single $c^{+}$ domain $\left(t_{\mathrm{ch}} \sim 4.6 \times 10^{5} \mathrm{~s}\right.$, scenario (1)) and slowest in single $c$ - domain $\left(t_{\mathrm{ch}}>2 \times 10^{6} \mathrm{~s}\right.$, scenario (2)). Clearly, the polarization accelerates the degradation process when it is in the field direction $(+z)$. Similarly the leakage currents in “ $\uparrow \backslash \rightarrow \uparrow$ ” type of $90^{\circ}$ domain structure $\left(t_{\mathrm{ch}} \sim 7.7 \times 10^{5} \mathrm{~s}\right.$, scenario (3)) increase faster than in “ $\downarrow \leftarrow \downarrow$ ” type of $90^{\circ}$ domain structure $\left(t_{\mathrm{ch}} \sim 1.28 \times 10^{6} \mathrm{~s}\right.$, scenario (4)). This is because the trend of the polarization direction isstill along the applied field direction in (3) and reverses to the field direction in (4). Notably, $t_{\mathrm{ch}}$ in (3) is also slower than that in (1), since domain structure of (3) consists of half $a+$ and $c^{+}$domain types, with only $c^{+}$contributing to the current evolution and the rest $a+$ less interacted with the ionic transport. The charged $90^{\circ}$ domain walls in (3) could alsoimpede the oxygen vacancy migration and,consequently, the leakage current increase. Conversely, the current increases faster in (4) compared to (2), since the former structure consists of only half $c$ - domain that is known to inhibit current increase.Finally, the multi-domain structure is considered a combination of all possible a/c domains and $180^{\circ} / 90^{\circ}$ domain walls; therefore, the $t_{\mathrm{ch}}$ is expected to be average of the other four scenarios $\left(t_{\mathrm{ch}} \sim 9.4 \times 10^{5} \mathrm{~s}\right)$. 


\section{Conclusions}

In this work, we developed a phase-field model combined with transport equations to study the space charge distribution, ionic transport, electrostatic evolution, and resistance degradationof a preset multi-domain structure $\mathrm{BaTiO}_{3}$ single plate ferroelectric capacitor.Our results showthat polarization induced bound charges along $90^{\circ}$ domain wallsaccount for the local oxygen vacancysegregation, while the $180^{\circ}$ domain walls and domain interiors remain charge neutral and have less influence on the defect distribution. Upon an external DC bias, remarkable electric potential gradient and potential barrier, and the resulting local electric field concentration, are observable at the $90^{\circ}$ charged domain walls, which partially blocks the oxygen vacancies migration, resulting in a local ionic enrichment and depletion on either side of the $90^{\circ}$ domain walls. On the other hand, the effect of $180^{\circ}$ domain walls on local electrostatics and oxygen vacancy evolution is hardly seen. The influence of multi-domain structure onthe resistance degradation behavior under room temperature is further investigated by comparing the leakage current evolutions and degradation characteristic time $\left(t_{\mathrm{ch}}\right)$ among different possible domain configurations in $\mathrm{BaTiO}_{3}$.

\section{Acknowledgements}

The work at Penn State was supported by U.S. Department of Defense, Air Force under FA9550-14-1-0264 (Chen) and FA9550-14-1-0067 (Randall), and theNational Science Foundation as part of the IUCRC Center for Dielectrics and Piezoelectrics under Grant No. IIP1361571 at Penn State. The work of Shen is partially supported by NSF DMS-1215066 and by the Computational Materials and Chemical Sciences Network (CMCSN) 
Table 1, Parameters used in the simulation

\begin{tabular}{llll}
\hline Parameter & Value & Parameter & Value \\
\hline Temperature, $T(\mathrm{~K})$ & 298 & Conduction band, $E_{c}(\mathrm{eV})[19]$ & -3.6 \\
Total thickness, $L(\mathrm{~nm})$ & 500 & Valence band, $E_{v}(\mathrm{eV})$ & -6.7 \\
External applied bias $(\mathrm{V})$ & 0.5 & $\begin{array}{l}\text { Work function of Ni electrode, } \\
E_{f m}(\mathrm{eV})\end{array}$ & -5.2 \\
& & $\begin{array}{l}\text { Effective Density of States, } N_{\mathrm{c}}, \\
\text { Oxygen vacancy mobility, }\end{array}$ & $10^{22}$ \\
$\mu_{\mathrm{Vo}} \cdot\left(\mathrm{cm}^{2} \cdot \mathrm{V}^{-1} \cdot \mathrm{s}^{-1}\right)[7]$ & $1.0 \times 10^{-14}$ & & \\
\hline
\end{tabular}

\section{Figure Captions:}

Fig. 1.2D plot of the equilibrium state of (a) domain structure, (b) polarization induced bound charges $\left(i=1 \sim 3\right.$ ), (c) oxygen vacancy distribution, (d) electric potential distribution and (e) shear stress $\sigma_{5}$. (f) 1D comparison of spacecharge (solid lines) and polarization charge (dashed lines) distributions along particular directions as indicated by the red and green dashed lines in (a). The red and green ordinates in (f) (note with different scales) are for charge distributions along direction (1) and (2) respectively as indicated in (a).

Fig. 2.1D plot of oxygen vacancy evolution along $z$ direction in each of the $1 / 4$ of the entire multi-domain $\mathrm{BaTiO}_{3}$ under $\mathrm{U}_{\mathrm{DC}}=0.5 \mathrm{~V}$ at room temperature: (a) $x / \mathrm{H}=0.0 \sim 0.5$; (b) $x / \mathrm{H}=0.5 \sim 1.0$; (c) $x / \mathrm{H}=1.0 \sim 1.5$; (d) $x / H=1.5 \sim 2.0$. $(\mathrm{H}=250 \mathrm{~nm}$ is half of the film thickness, so that $\mathrm{z} / \mathrm{H}$ from -1 to +1 represents the entire film thickness)

Fig. 3.1D plot of electric potential (black solid lines) and electric field (red solid lines) distributions at $\mathrm{t}=2.5 \times 10^{6} \mathrm{~s}$ along $\mathrm{z}$ direction in each of the $1 / 4$ of the entire multi-domain $\mathrm{BaTiO}_{3}$ under $\mathrm{U}_{\mathrm{DC}}=0.5 \mathrm{~V}$ at room temperature: (a) $x / \mathrm{H}=0.0 \sim 0.5$; (b) $x / \mathrm{H}=0.5 \sim 1.0$; (c) $x / \mathrm{H}=1.0 \sim 1.5$; (d) $x / \mathrm{H}=1.5 \sim 2.0$. The light blue arrows indicate the domain orientations. Dark blue dashed lines indicate the positions of $180^{\circ}$ domain walls, green dashed lines indicate the positions of $90^{\circ}$ domain walls $(\downarrow \leftarrow \downarrow)$ whereelectric potential locally drops, and red dashed lines indicate the positions of $90^{\circ}$ domain walls $(\uparrow \backslash \rightarrow \mid \uparrow)$ where there is local electric potential barrier blocking the ionic transport.

Fig. 4. Illustration of interactions between polarization charges, ionic transport, and electric potential/field by replottingFig. 1(f), Fig. 2(b) and Fig. 3(b) into Fig. 4(a) (c). The electric potential drop at the $90^{\circ}$ domain wall (green dashed lines) induces local positive electric field to facilitate the oxygen vacancy drift (c), and counteracts the oxygen vacancy diffusion in the opposite direction due to local concentration gradient (b), which is induced by the positive/negative polarization charges aside the $90^{\circ}$ domain wall (a). 
Fig. 5. (a) Schematic plot of possible domain configurations of $\mathrm{BaTiO}_{3}$ and (b) comparison of current density evolution of $\mathrm{BaTiO}_{3}$ capacitor of different domain configurations at $25^{\circ} \mathrm{C}$ under $\mathrm{U}_{\mathrm{DC}}=0.5 \mathrm{~V}$. The colors of the schematic domain configurations in (a) correspond to the colors of the current density evolution in (b). The "+" mark the degradation characteristic time $\left(t_{\mathrm{ch}}\right)$ for different domain configurations.

\section{References:}

[1] H. Kishi, Y. Mizuno, H. Chazono. Base-metal electrode-multilayer ceramic capacitors: Past, present and future perspectives, Jpn J Appl Phys 142 (2003) 1-15.

[2] A.V. Polotai, A.V. Ragulya, C.A. Randall. Preparation and size effect in pure nanocrystalline barium titanate ceramics, Ferroelectrics 288 (2003) 93-102.

[3] R. Waser, T. Baiatu, K.H. Hardtl. Dc Electrical Degradation of Perovskite-Type Titanates .1. Ceramics, J Am Ceram Soc 73 (1990) 1645-1653.

[4] S. Zhao, S.J. Zhang, W. Liu, N.J. Donnelly, Z. Xu, C.A. Randall. Time dependent de resistance degradation in lead-based perovskites: $0.7 \mathrm{~Pb}\left(\mathrm{Mg}_{1 / 3} \mathrm{Nb}_{2 / 3}\right) \mathrm{O}_{3}-0.3 \mathrm{PbTiO}_{3}, \mathrm{~J}$ Appl Phys 105 (2009) 053705.

[5] C.A. Randall, R. Maier, W. Qu, K. Kobayashi, K. Morita, Y. Mizuno, N. Inoue, T. Oguni. Improved reliability predictions in high permittivity dielectric oxide capacitors under high dc electric fields with oxygen vacancy induced electromigration, J Appl Phys 113 (2013) 014101.

[6] R. Waser, T. Baiatu, K.H. Hardtl. Dc Electrical Degradation of Perovskite-Type Titanates .2. Single-Crystals, J Am Ceram Soc 73 (1990) 1654-1662.

[7] H.I. Yoo, M.W. Chang, T.S. Oh, C.E. Lee, K.D. Becker. Electrocoloration and oxygen vacancy mobility of $\mathrm{BaTiO}_{3}$, J Appl Phys 102 (2007) 093701.

[8] W. Liu, G.Y. Yang, C.A. Randall. Evidence for Increased Polaron Conduction Near the Cathodic Interface in the Final Stages of Electrical Degradation in $\mathrm{SrTiO}_{3}$ Crystals, Jpn J Appl Phys 48 (2009) 051404.

[9] W. Liu, C.A. Randall. Thermally Stimulated Relaxation in Fe-Doped $\mathrm{SrTiO}_{3}$ Systems: I. Single Crystals, J Am Ceram Soc 91 (2008) 3245-3250.

[10] W. Liu, C.A. Randall. Thermally Stimulated Relaxation in Fe-Doped SrTiO3 Systems: II. Degradation of $\mathrm{SrTiO}_{3}$ Dielectrics, J Am Ceram Soc 91 (2008) 3251-3257.

[11] G.Y. Yang, G.D. Lian, E.C. Dickey, C.A. Randall, D.E. Barber, P. Pinceloup, M.A. Henderson, R.A. Hill, J.J. Beeson, D.J. Skamser. Oxygen nonstoichiometry and dielectric evolution of $\mathrm{BaTiO}_{3}$. Part II - insulation resistance degradation under applied dc bias, J Appl Phys 96 (2004) 7500-7508.

[12] T. Baiatu, R. Waser, K.H. Hardtl. Dc Electrical Degradation of Perovskite-Type Titanates .3. A Model of the Mechanism, J Am Ceram Soc 73 (1990) 1663-1673.

[13] T. Holbling, N. Soylemezoglu, R. Waser. A mathematical-physical model for the charge transport in p-type $\mathrm{SrTiO}_{3}$ ceramics under dc load: Maxwell-Wagner relaxation, Journal of Electroceramics 9 (2002) 89-102.

[14] R. Meyer, R. Liedtke, R. Waser. Oxygen vacancy migration and time-dependent leakage current behavior of $\mathrm{Ba}_{0.3} \mathrm{Sr}_{0.7} \mathrm{TiO}_{3}$ thin films, Appl Phys Lett 86 (2005) 112904.

[15] Y. Cao, J. Shen, C. Randall, L.Q. Chen. Effect of Ferroelectric Polarization on Ionic Transport and Resistance Degradation in $\mathrm{BaTiO}_{3}$ by Phase-Field Approach, J Am Ceram Soc 97 (2014) 3568-3575.

[16] D. Damjanovic. Ferroelectric, dielectric and piezoelectric properties of ferroelectric thin films and ceramics, Rep Prog Phys 61 (1998) 1267-1324.

[17] D. Damjanovic. Stress and frequency dependence of the direct piezoelectric effect in ferroelectric ceramics, J Appl Phys 82 (1997) 1788-1797.

[18] Y. Xiao, K. Bhattacharya. Interaction of oxygen vacancies with domain walls and its impact on fatigue in ferroelectric thin films, Smart Structures and Materials 2004: Active Materials: Behavior and Mechanics 5387 (2004) 354-365. 
[19] Y. Xiao, V.B. Shenoy, K. Bhattacharya. Depletion layers and domain walls in semiconducting ferroelectric thin films, Phys Rev Lett 95 (2005) 247603.

[20] Q.S. Zhang, W.A. Goddard. Charge and polarization distributions at the 90 degrees domain wall in barium titanate ferroelectric, Appl Phys Lett 89 (2006) 182903.

[21] J. Hlinka, P. Marton. Phenomenological model of a 90 degrees domain wall in $\mathrm{BaTiO}_{3}$-type ferroelectrics, Phys Rev B 74 (2006) 104104.

[22] C. Korte, N. Schichtel, D. Hesse, J. Janek. Influence of interface structure on mass transport in phase boundaries between different ionic materials Experimental studies and formal considerations, Monatsh Chem 140 (2009) 1069-1080.

[23] N. Schichtel, C. Korte, D. Hesse, J. Janek. Elastic strain at interfaces and its influence on ionic conductivity in nanoscaled solid electrolyte thin films-theoretical considerations and experimental studies, Phys Chem Chem Phys 11 (2009) 3043-3048.

[24] H.L. Hu, L.Q. Chen. Computer simulation of 90 degrees ferroelectric domain formation in twodimensions, Materials Science and Engineering a-Structural Materials Properties Microstructure and Processing 238 (1997) 182-191.

[25] Y.L. Li, S.Y. Hu, Z.K. Liu, L.Q. Chen. Phase-field model of domain structures in ferroelectric thin films, Appl Phys Lett 78 (2001) 3878-3880.

[26] Y.L. Li, S.Y. Hu, Z.K. Liu, L.Q. Chen. Effect of substrate constraint on the stability and evolution of ferroelectric domain structures in thin films, Acta Mater 50 (2002) 395-411.

[27] Y.L. Li, L.Q. Chen, G. Asayama, D.G. Schlom, M.A. Zurbuchen, S.K. Streiffer. Ferroelectric domain structures in $\mathrm{SrBi}_{2} \mathrm{Nb}_{2} \mathrm{O}_{9}$ epitaxial thin films: Electron microscopy and phase-field simulations, $\mathrm{J}$ Appl Phys 95 (2004) 6332-6340.

[28] Y.L. Li, L.E. Cross, L.Q. Chen. A phenomenological thermodynamic potential for $\mathrm{BaTiO}_{3}$ single crystals, J Appl Phys 98 (2005) 064101.

[29] F.A. Kroger, H.J. Vink. Relations between the Concentrations of Imperfections in Crystalline Solids, Solid State Phys 3 (1956) 307-435.

[30] L.Q. Chen, J. Shen. Applications of semi-implicit Fourier-spectral method to phase field equations, Comput Phys Commun 108 (1998) 147-158.

[31] Y. Cao, S. Bhattacharya, J. Shen, C.A. Randall, L.Q. Chen. Role of polaron hopping in leakage current behavior of $\mathrm{SrTiO}_{3}$ single crystal, Journal of Applied Physics 114 (2013) 224102. 


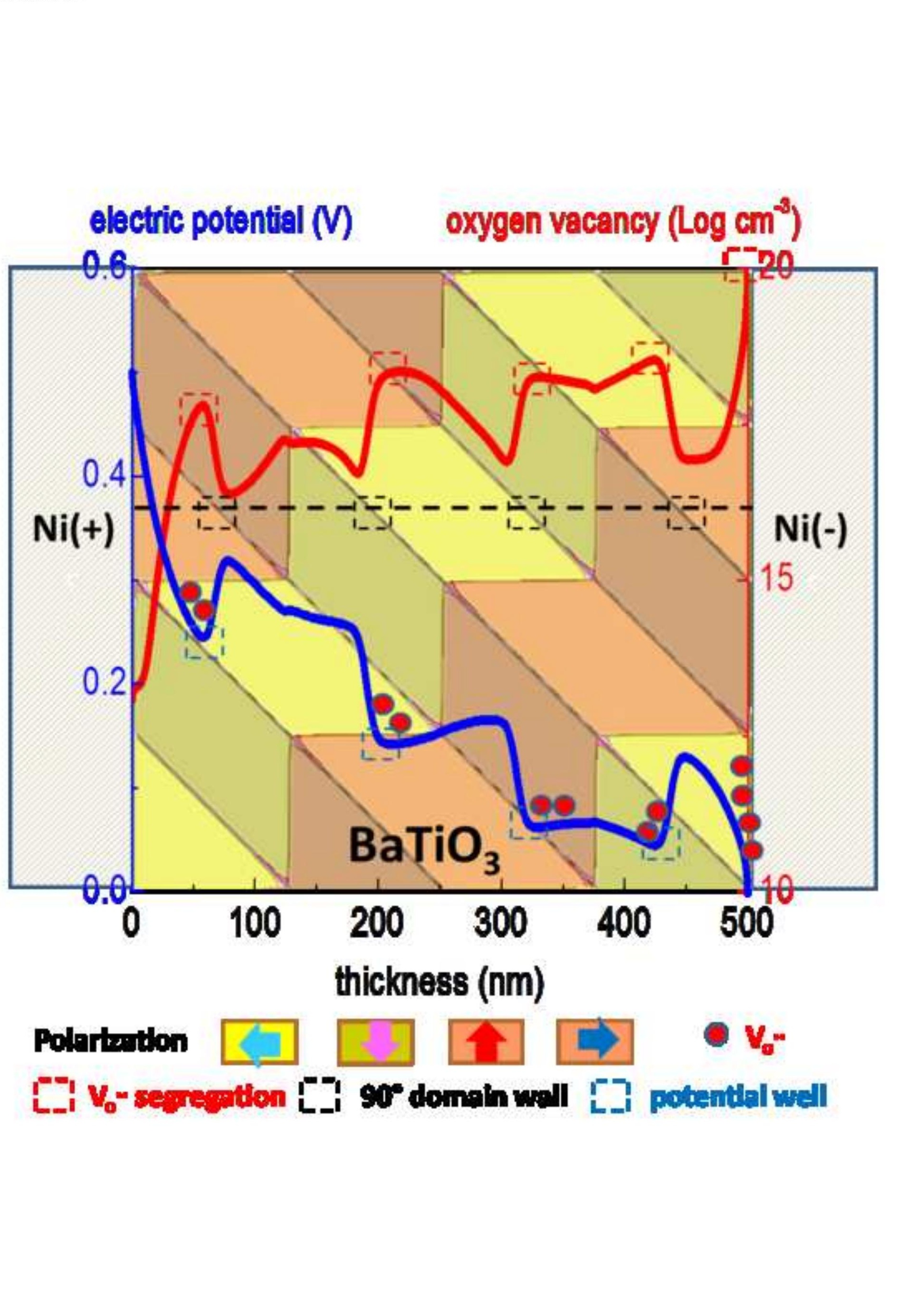

Polartuition
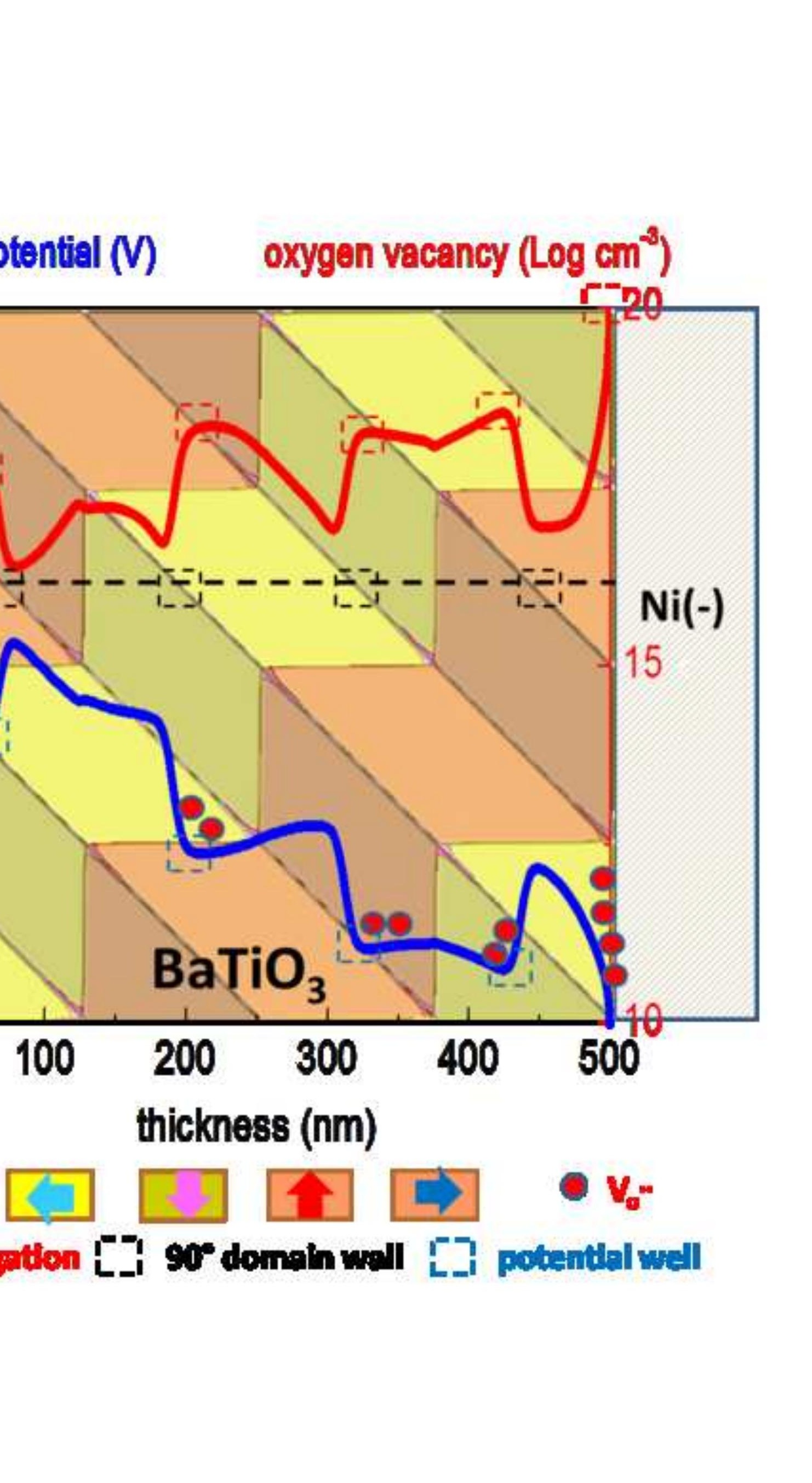

- $v_{\mathrm{g}}$

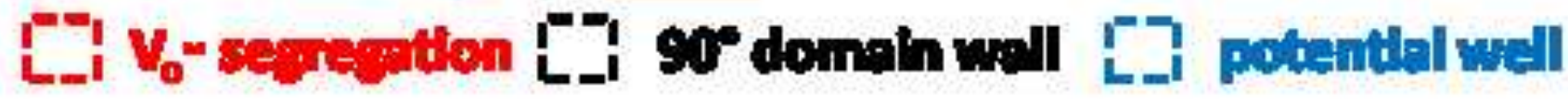

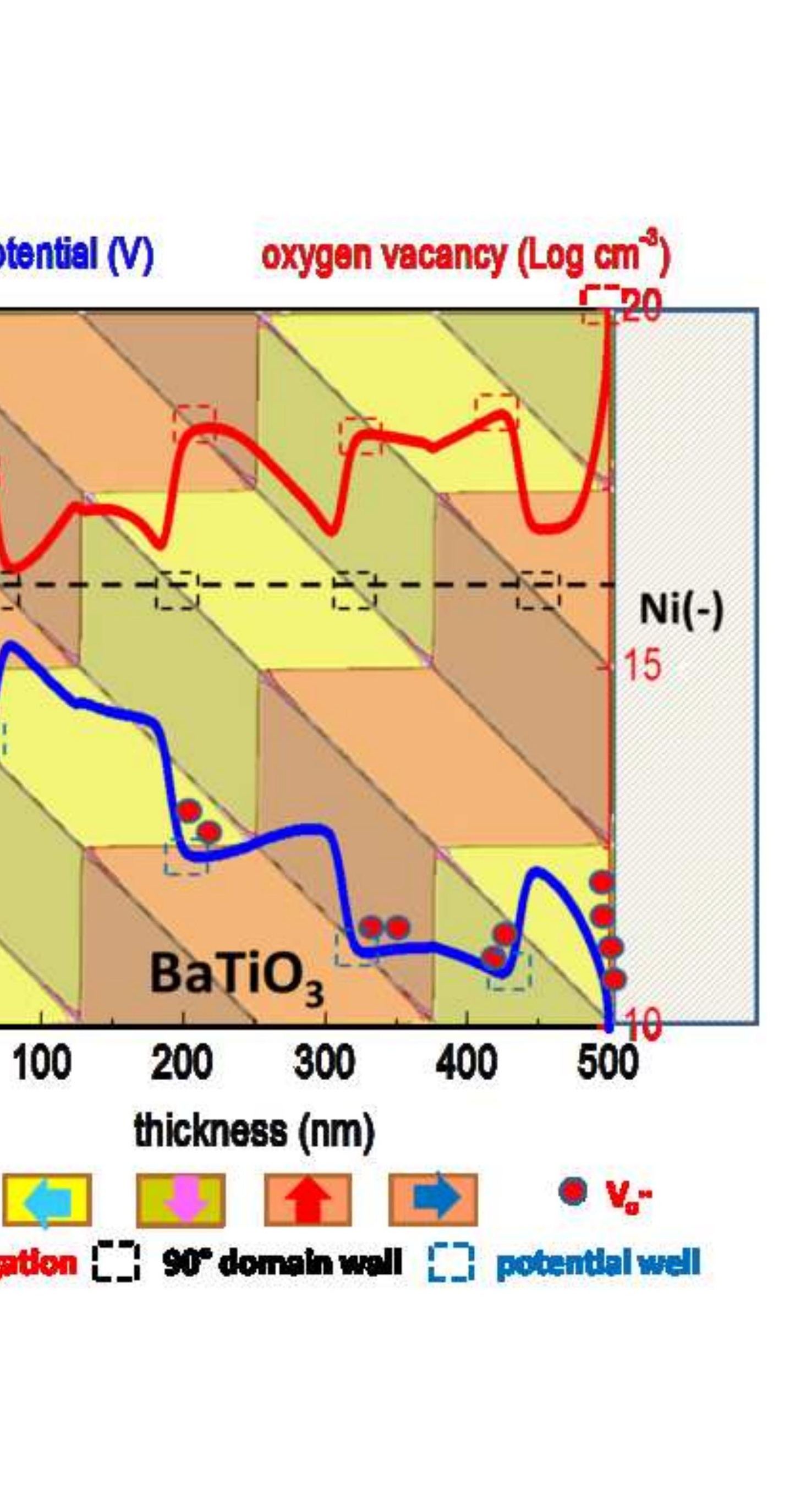

.


(a) $\quad-\mathrm{dP}_{\mathrm{i}} / \mathrm{dx}_{\mathrm{i}}\left(\mathrm{C} / \mathrm{cm}^{3}\right)$
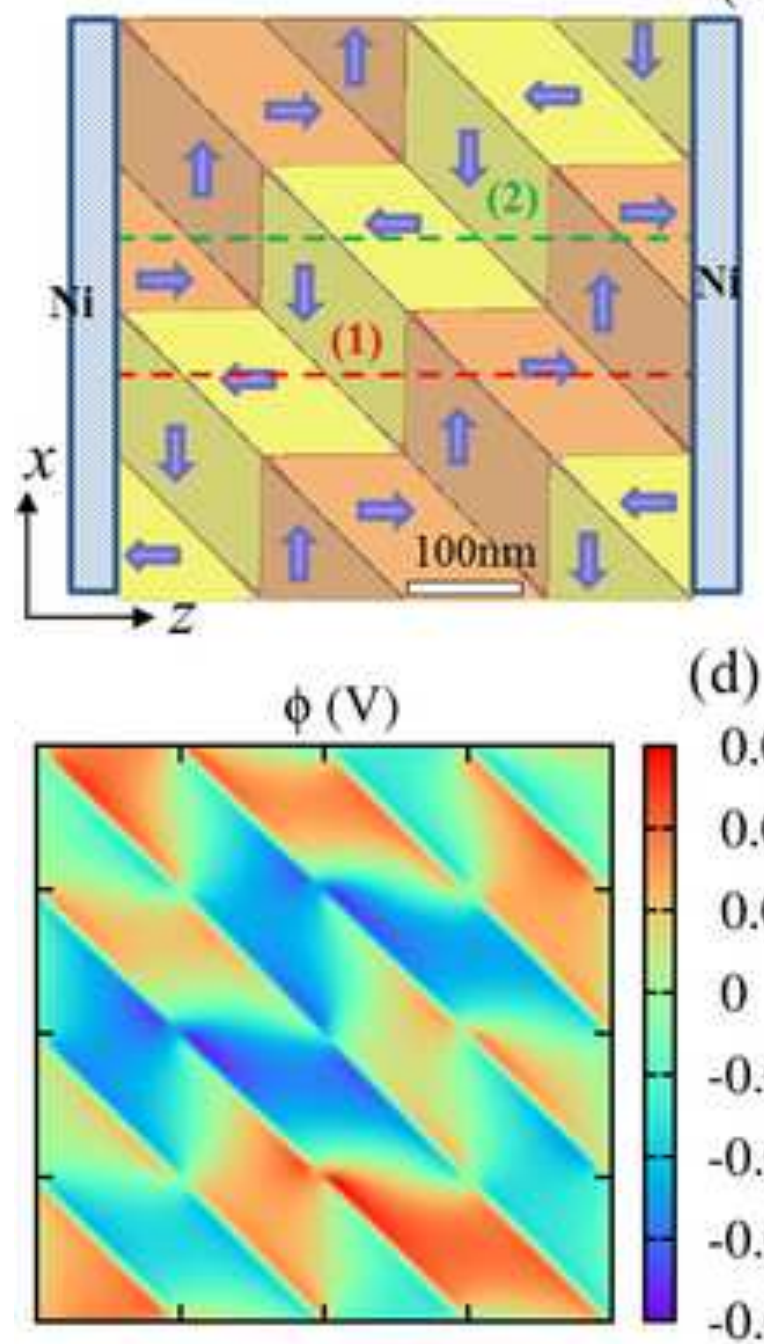

(d)

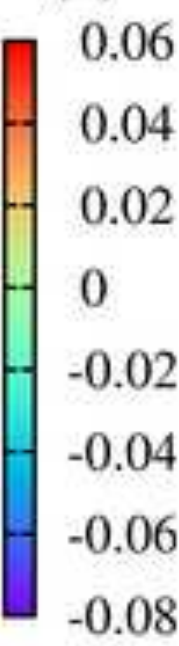

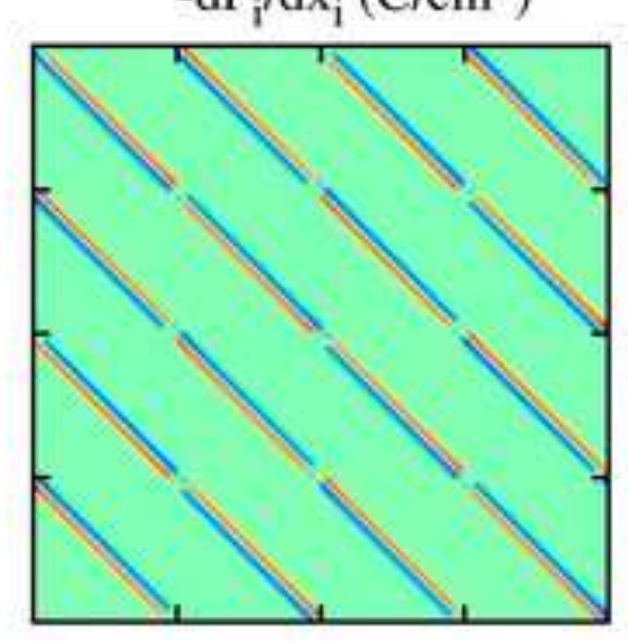

$\sigma_{5}(\mathrm{GPa})$

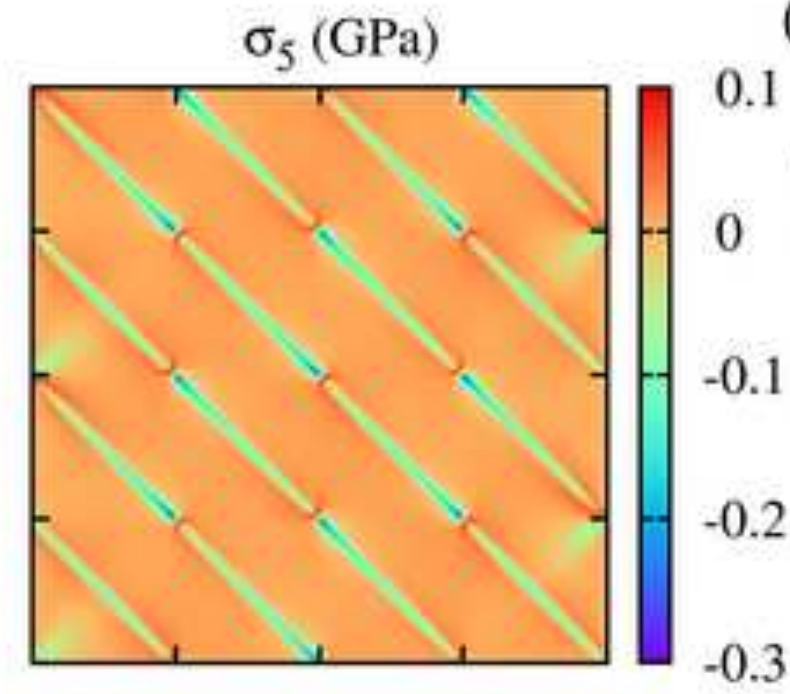

$5^{(b)}$

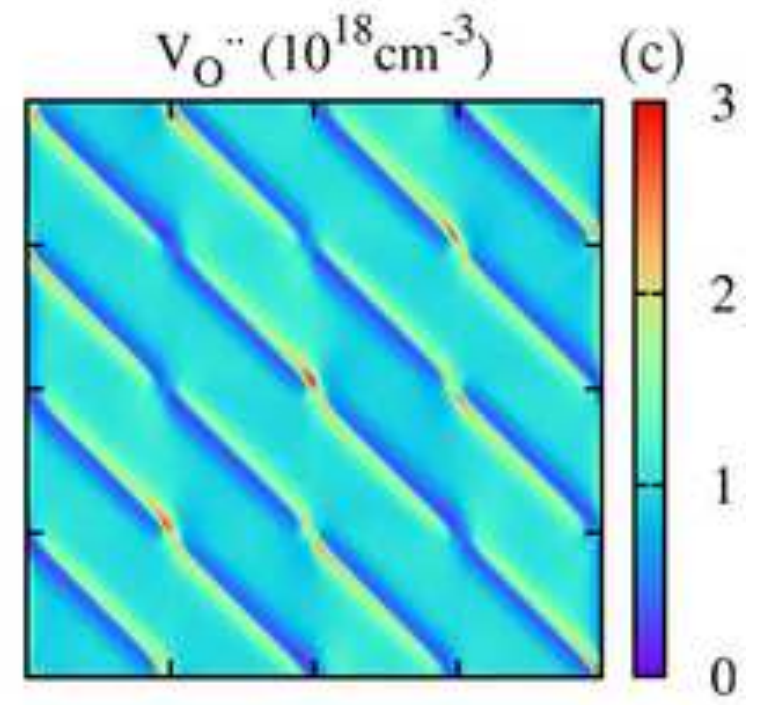

(e)

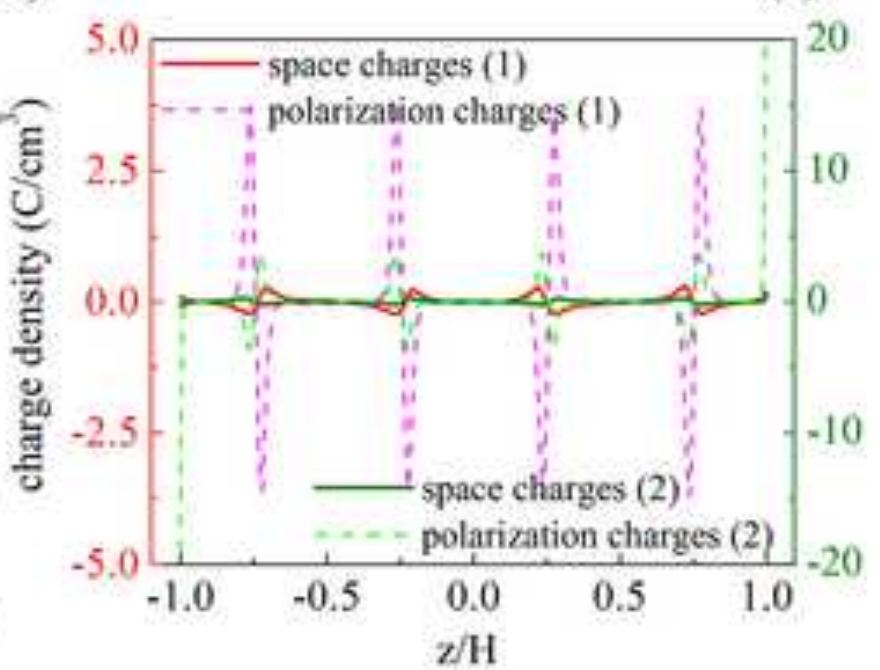

(f)

0 

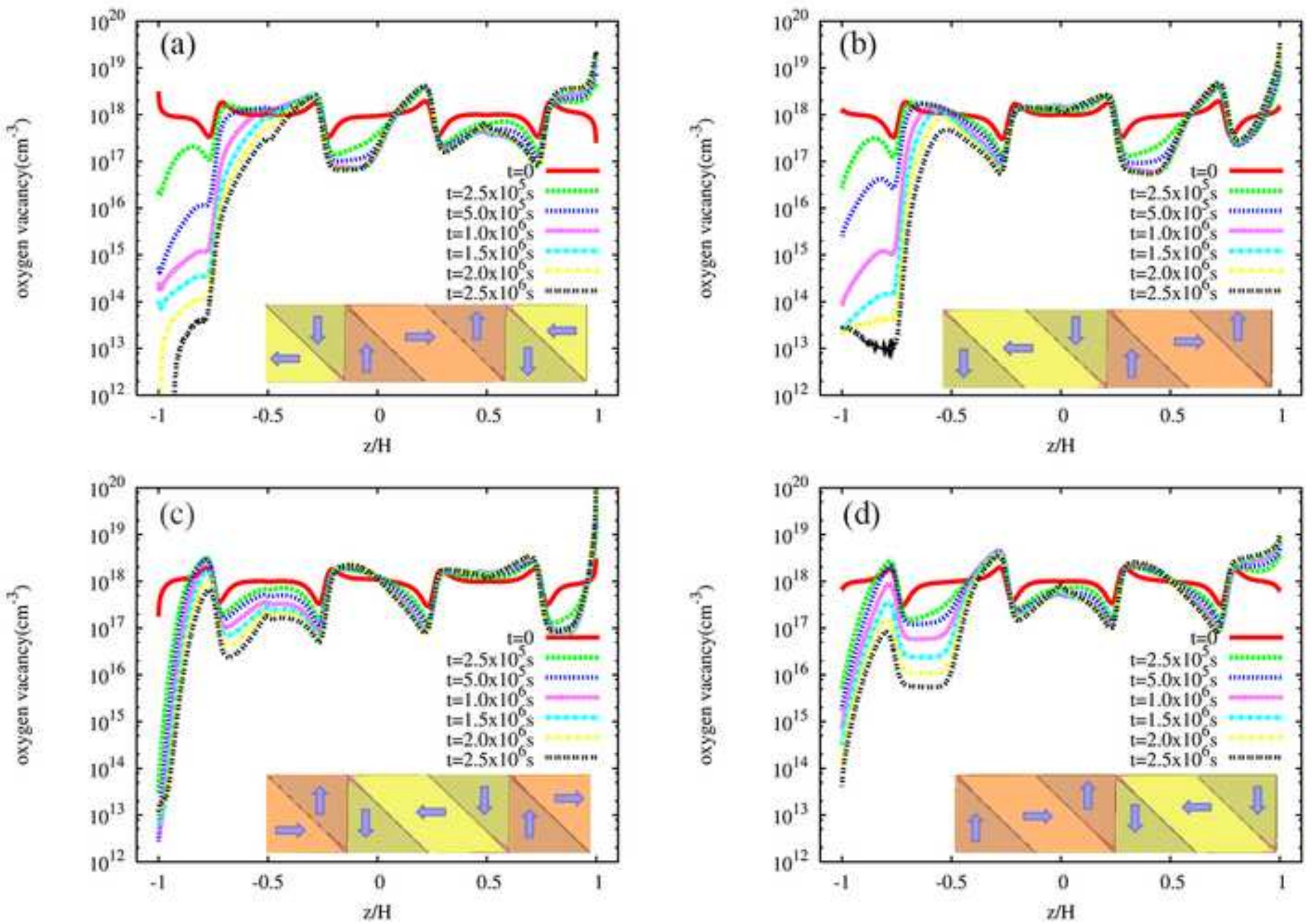

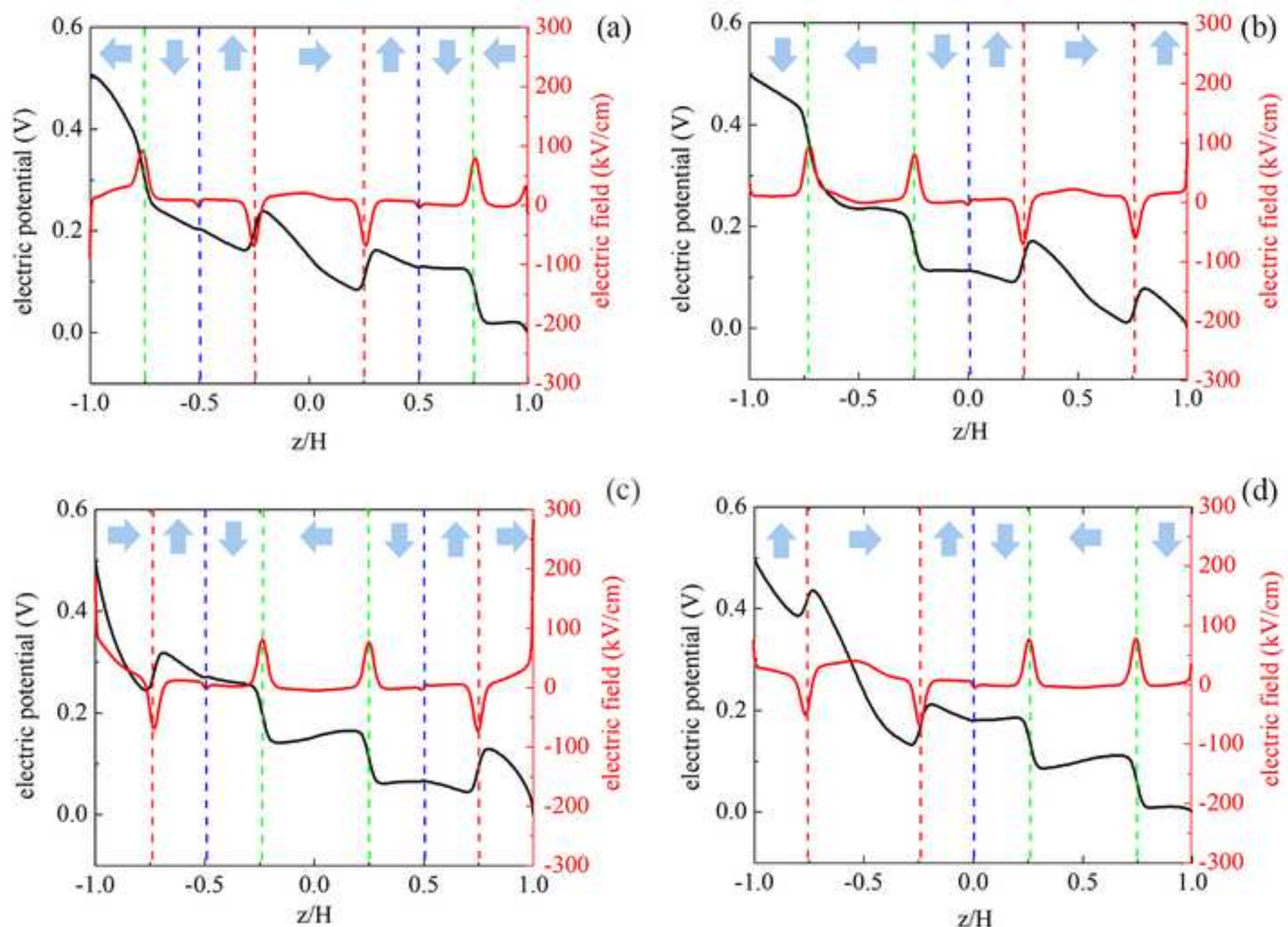

(c)

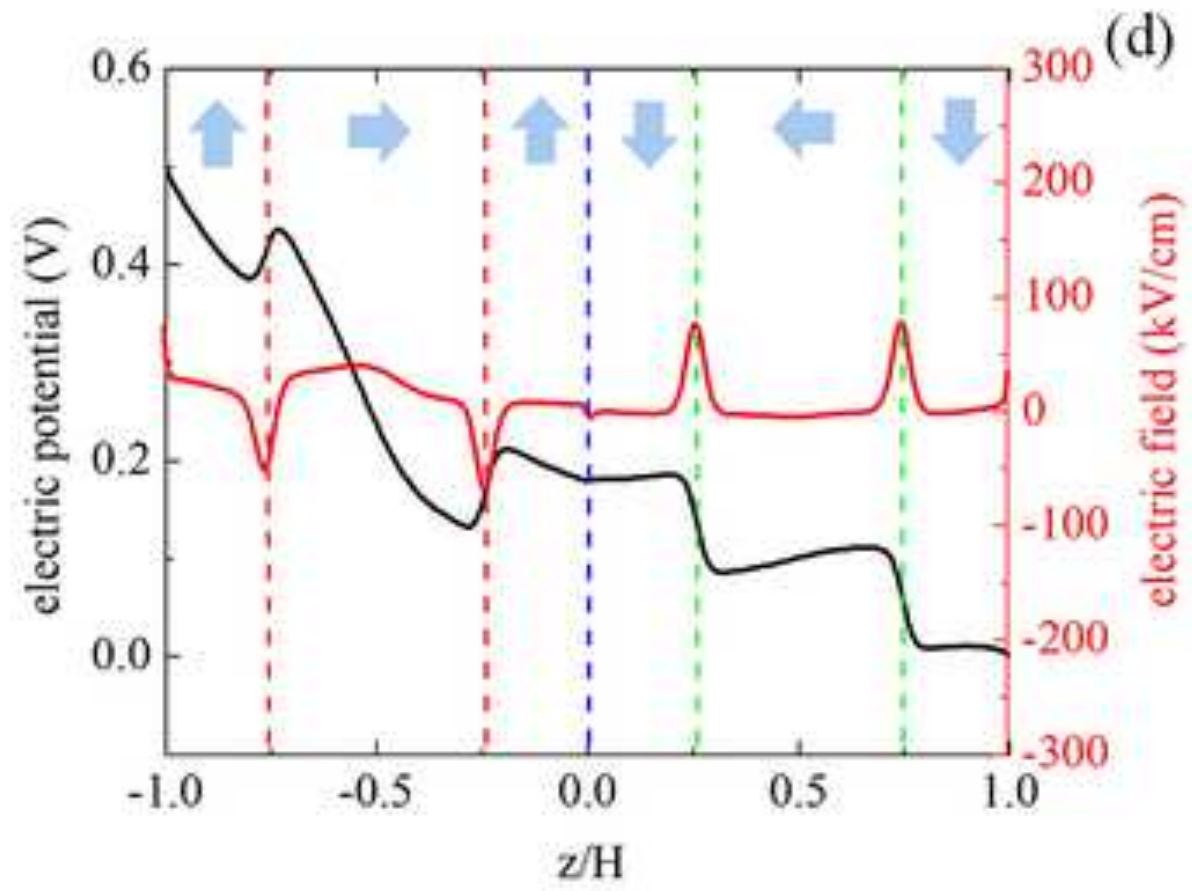

톨




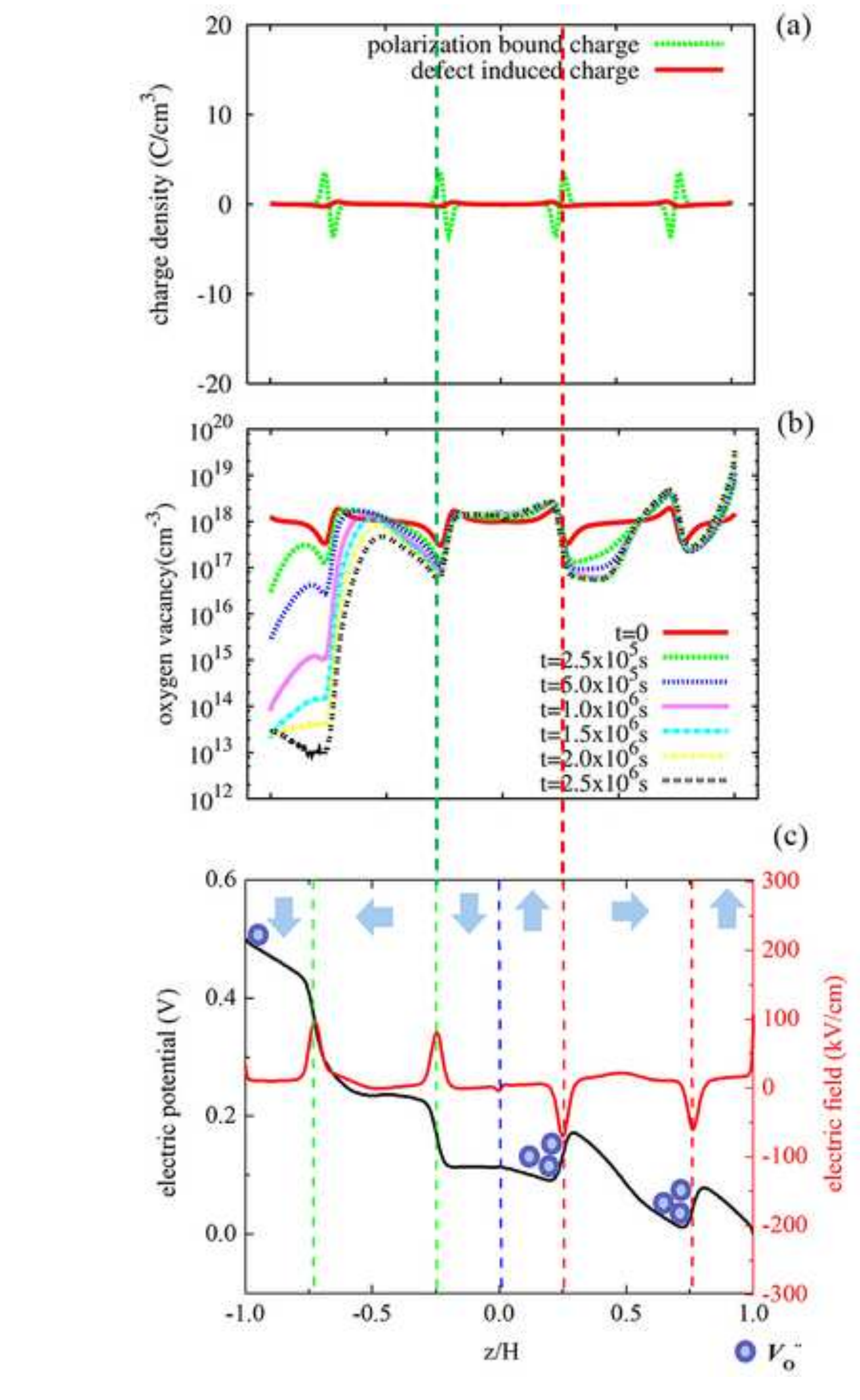

Figure 4

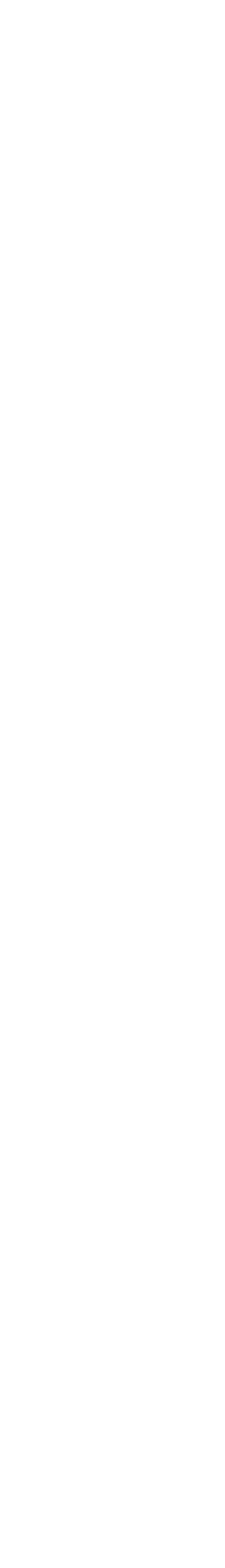
4

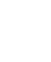


(a)

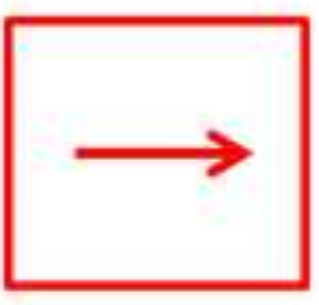

(1)

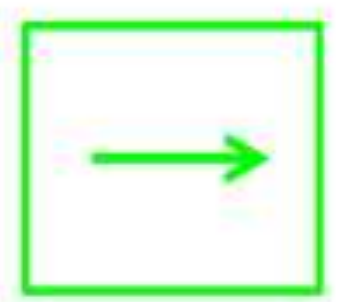

(2)

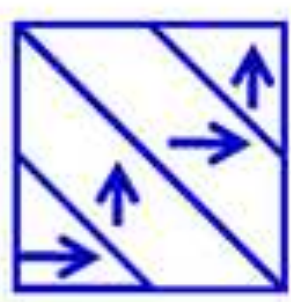

(3)

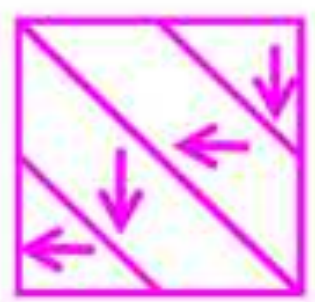

(4)

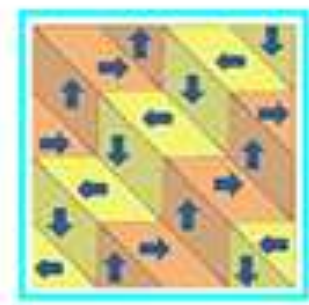

(5)

(b)

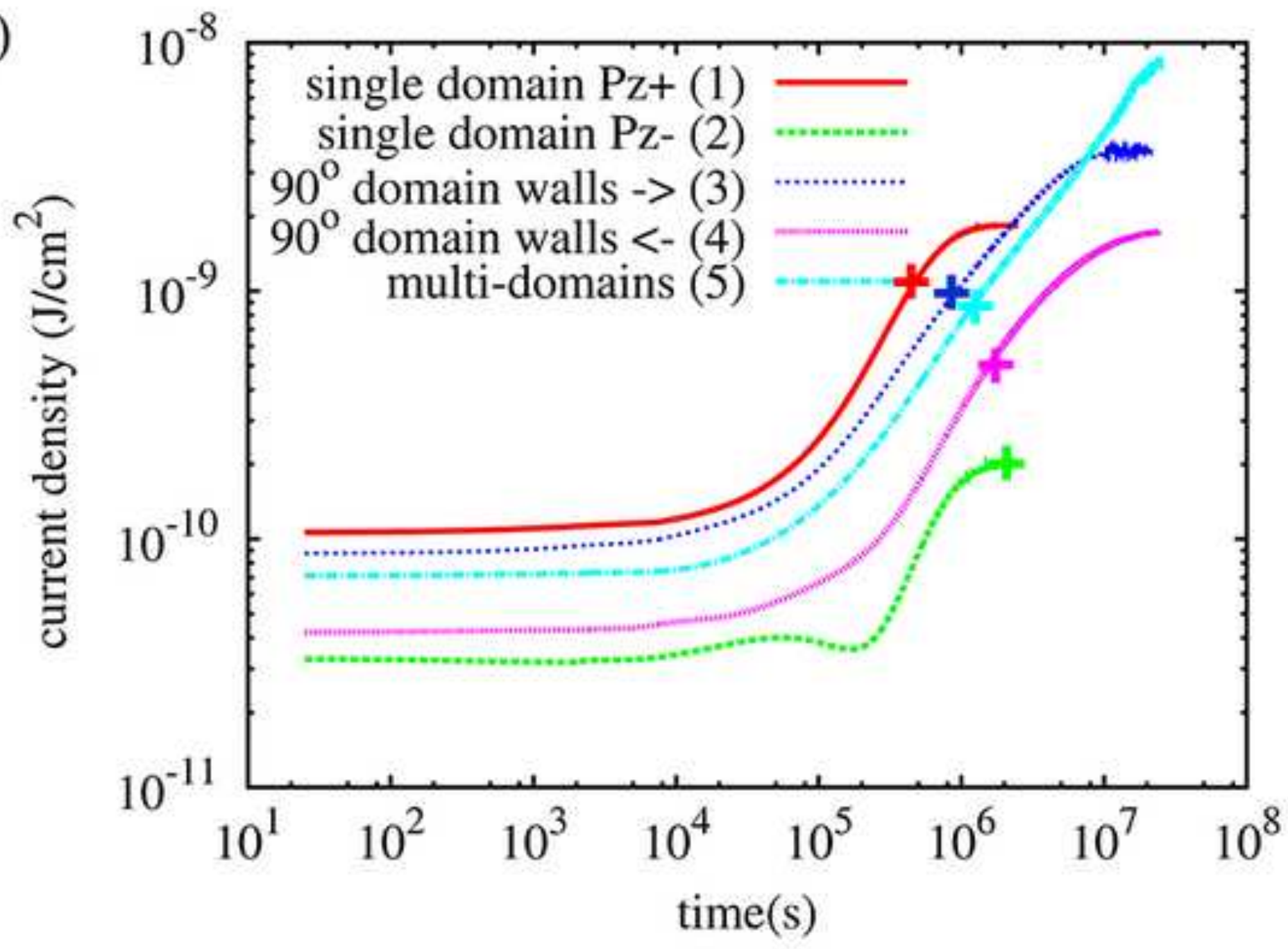

